\title{
The general equations of biological strife in the case of historical actions
}

\author{
By Vito Volterra. \\ (Received 4th July, 1938. Read 4th November, 1938.)
}

1. I have begun to study the laws of the struggle for life by a group of species living in the same environment in such a way that some devour others. I have used for this purpose the so called principle of encounter, considering the encounters of the individuals of the various species, and what follows by reason of the actions that the individuals exercise on one another ${ }^{1}$.

Making very simple and probable hypotheses, the equations to which it leads are the following:

$$
\beta_{r} \frac{d N}{d t} r=\left(\epsilon_{r} \beta_{r}+\sum_{1}^{n} a_{s r} N_{s}\right) N_{r}, \quad\left(a_{r s}=-a_{s r}\right)
$$

in which $N_{1}, N_{2}, \ldots N_{n}$ denote the populations of the various species, $\epsilon_{1}, \epsilon_{2}, \ldots \epsilon_{n}$ the coefficients of auto-increase of the various species and $\beta_{1}, \beta_{2}, \ldots \beta_{n}$ the values of the individuals.

These equations are well known and their consequences can be summed up in the following general laws:

LAw I. Law of conservation of fluctuations.

The populations of the different species are limited in positive numbers and there are always fluctuations that never die out.

LAW II. Law of conservation of means.

If we take as means of the different species the asymptotic means they are constant and independent of the initial populations.

LAw III. Laws of perturbation of means.

If all the species are destroyed uniformly and in proportion to the numbers of their individuals, there are always some species which

1 Variazioni e fluttuarioni del numero di individui un specie animali convirenti. Memorie della R. Accademia dei Lincei S. 6, 2 (1926), 31-113. 
profit and others which suffer. Among the first, there is at least one which is devoured by the others and among the second there is also one species that devours others.

2. But in this treatment only immediate actions are considered. Now in all biological phenomena it is necessary to examine not only immediate actions but also those depending on the past, that is, on the changes which the species have undergone. These actions were first called hereditary actions; but this name was not well chosen, although it may have been useful to signify such phenomena in the inorganic world. It was found preferable to use the term historical actions or actions belonging to memory. We shall use the first of these two denominations.

To treat this case I have tried to extend the method of encounter which has already been happily employed; in the case of two species, one of which devours the other, the same method was perfectly successful and the three general laws could be extended, introducing only a few modifications.

For this consult the work: Leçons sur la théorie mathématique de la lutte pour la vie. Paris, Gauthier-Villars, 1931.

But if we are determined to extend the same method to any number of species living together there arise difficulties which seem not easily resolvable.

Shall we then give up the study of a general case taking the historical phenomenon into account? Or will it be possible to change the method, no longer using the method of encounter proved useful ?

\section{II}

3. Last year, at the Mathematical Society of Francer, I gave a lecture in which I examined only the case of immediate actions, but did not follow the method of encounters. I used instead a more simple and intuitive, but perhaps not so rigorous, method. I believe that this method can be easily applied to the historical case not only of two species, but also to that of any number of species.

This method consists fundamentally in making all the actions exercised in the past, that is, all the historical actions, enter into the values of the coefficients of increase.

1 Fluctuations dans la lutte pour la vie, leurs lois fondamentales et de reciprocité. Conférence de la réunion internationale des mathématiciens, Paris 1938. 
4. After these general considerations let us expound the matter in detail. Assume that we have $n$ species whose populations are denoted by

$$
N_{1}, N_{2}, \ldots N_{n}
$$

If their coefficients of increase are called $\epsilon_{1}, \epsilon_{2}, \ldots \epsilon_{n}$, taking these constants as positive or negative according to whether the species tend to increase or to die out when not interfered with, we shall have the following equations expressing the variations of the populations:

$$
\frac{d N_{1}}{d t}=\epsilon_{1} N_{1}, \quad \frac{d N_{2}}{d t}=\epsilon_{2} N_{2}, \ldots \quad \frac{d N_{n}}{d t}=\epsilon_{n} N_{n}
$$

But we want to suppose that the various species act on one another dependently upon the number of these populations; and if we suppose this dependency to be of an immediate and linear nature, we shall have to substitute for $\epsilon_{1}, \epsilon_{2}, \ldots \epsilon_{n}$

$$
\epsilon_{1}+\sum_{1}^{n} A_{s 1} N_{s}, \quad \epsilon_{2}+\sum_{1}^{n} A_{s 2} N_{s}, \ldots \epsilon_{n}+\sum_{1}^{n} A_{s n} N_{s} .
$$

The coefficient $A_{s r}$ measures that unitary action (per individual) which the species $s$ exercises upon the species $r$, while $A_{r s}$ denotes the inverse action that species $r$ exercises upon the species $s$; and as it is supposed that these actions are such that, while one species injures the other, the latter profits from the first (for example, one species devours the other) the coefficients $A_{s r}, A_{r s}$ may be assumed to have opposite signs. They will not however be of equal absolute value.

Without repeating the discussion made in last year's lecture, we shall make a simple and probable hypothesis; that their absolute values be in the inverse ratio of certain constant and positive coefficients, which we shall assume as values of the individuals of each species. In this way we can write:

$$
A_{r s}=\frac{1}{\beta_{r}} a_{r s}, \quad A_{s r}=\frac{1}{\beta_{s}} a_{s r}, \quad a_{r \delta}=-a_{s r} ;
$$

and we shall have as general equations of the struggle for life the equations $(A)$, that is, the very same equations which are found by the method of encounters.

5. We can generalise further and suppose that the equations ( 1 ) do not hold and that the condition $A_{r r} \gtrless 0$ is satisfied, when instead of supposing that the individuals of the various species devour each other, we assume that they act on one another in such a way that 
they influence the generic coefficients of increase of the populations. Then we find the following equations

$$
\frac{d N_{r}}{d t}=\left(\epsilon_{r}+\sum_{1}^{n} A_{s r} N_{s}\right) N_{r}
$$

which have been classified and studied in paragraph 7, part II of "Variazioni e fluttuazioni del numero d'individui in specie animali conviventi." R. Comit. Talassografico Italiano, Mem. CXXXI, 1927.

\section{III}

6. Now we come to examine the historical case. By $t$ we denote the actual instant and by $\tau$ a preceding instant. The number of individuals of the species $s$ at time $\tau$ will be $N_{s}(\tau)$.

Let us suppose that the species $s$ exercises over the coefficient of increase of the species $r$ an action which will be manifested in the future and which varies with the distance in time. We shall denote such a (unitary) action by $F_{s r}(t-\tau)$ when it is exercised by the species $s$ in the infinitesimal interval of time $(\tau, \tau+d \tau)$ and is manifested on the species $r$ at time $t$. Then the action corresponding to the population $N_{s}(\tau)$ will be

$$
N_{s}(\tau) F_{s r}(t-\tau) d \tau
$$

If we take into account all these actions beginning from the origin of times at which they are supposed to have begun, up to the present moment $t$ we shall have

$$
\int_{0}^{t} N_{8}(\tau) F_{s r}(t-\tau) d \tau
$$

Considering historical actions for all $n$ species on the coefficient of increase of the species $r$ we shall have

$$
\sum_{1}^{n} \int_{0}^{t} N_{s}(\tau) F_{s r}(t-\tau) d \tau
$$

The coefficient of increase of the species $r$, taking into account all immediate and historical actions exercised upon it, will therefore become

$$
\epsilon_{r}+\sum_{1}^{n}\left(A_{s r} N_{s}(t)+\int_{0}^{t} N_{s}(\tau) F_{s r}(t-\tau) d \tau\right)
$$

And as general equations of the struggle for life we shall assume

$$
\frac{d N_{r}}{d t}=\left\{\epsilon_{r}+\sum_{s}^{n}\left[A_{s r} N_{s}(t)+\int_{0}^{t} N_{s}(\tau) F_{s r}(l-\tau) d \tau\right]\right\} N_{r}(t)
$$

In this way we shall have $n$ integro-differential equations. 
It is not impossible that some of the $A_{s r}, F_{s r}$ may be zero, and up to now we shall assume nothing about their signs.

7. We may suppose that the historical actions may be prolonged indefinitely in the past, and then the equations $(B)$ must be replaced by the following:

$$
\frac{d N_{r}}{d t}=\left\{\epsilon_{r}+\sum_{s}^{n}\left[A_{s r} N_{\varepsilon}(t)+\int_{-\infty}^{t} N_{s}(\tau) F_{s r}(t-\tau) d \tau\right]\right\} N_{r}(t) .
$$

Of course we must suppose that the necessary conditions for convergence of these integrals are satisfied. We may also suppose that the historical actions cease after a certain interval of time, and then it is sufficient to change the lower limit of the preceding integral into $t-T_{0}$, denoting by $T_{0}>0$ this interval of time. Then $\left(B^{\prime}\right)$ becomes

$$
\left(B^{\prime \prime}\right) \quad \frac{d N_{r}}{d t}=\left\{\epsilon_{r}+\sum_{1}^{n}\left[A_{s r} N_{s}(t)+\int_{t-T_{s}}^{t} N_{s}(\tau) F_{s r}(t-\tau) d \tau\right]\right\} N_{r}(t),
$$

or

$\left(B^{\prime \prime \prime}\right) \quad \frac{d N_{r}}{d t}=\left\{\epsilon_{r}+\sum_{1}^{n}\left[A_{s r} N_{s}(t)+\int_{0}^{T_{0}} N_{s}(t-\tau) F_{s r}(\tau) d \tau\right]\right\} N_{r}(t)$.

8. We easily see that there may be stationary states. For that purpose we shall substitute in the equations $\left(B^{\prime \prime \prime}\right)$ for $N_{1}, N_{2}, \ldots N_{n}$ the constants $K_{1}, K_{2}, \ldots K_{n}>0$.

The equations become

$$
\epsilon_{r}+\sum_{1}^{n}\left(A_{s r}+\int_{0}^{T_{\mathrm{o}}} \boldsymbol{F}_{s r}(\tau) d \tau\right) K_{s}=0
$$

and if we write

$$
A_{s r}+\int_{0}^{T_{0}} F_{s r}(\tau) d \tau=C_{s r}
$$

we shall have

$$
\epsilon_{r}+\sum_{1}^{n} C_{s r} K_{s}=0
$$

If the determinant of the $C_{s r}$ is different from 0 and the roots positive, these will represent the populations of equilibrium (stationary states).

9. Let us take again the equation $(B)$, multiply both sides by $\frac{d t}{N_{r}(t)}$ and then integrate between 0 and $\theta$. We find

$$
\log \frac{N_{r}(\theta)}{N_{r}(0)}=\epsilon_{r} \theta+\sum_{1}^{n} \int_{0}^{\theta}\left[A_{s r} N_{s}(t)+\int_{0}^{t} N_{s}(\tau) F_{s r}(t-\tau) d \tau\right] d t .
$$


Applying a well known Dirichlet's transformation, we obtain

$$
\log \frac{N_{r}(\theta)}{N_{r}(\overline{0})}=\epsilon_{r} \theta+\sum_{1}^{n} \int_{0}^{\theta}\left[A_{s r}+\int_{t}^{\theta} F_{s r}(\tau-t) d \tau\right] N_{s}(t) d t,
$$

from which it follows that

$$
N_{r}(\theta)=N_{r}(0) e^{p}
$$

where $\quad P=\epsilon_{r} \theta+\sum_{1}^{n} \int_{0}^{\theta}\left[A_{s r}+\int_{t}^{\theta} F_{s r}(\tau-\cdot t) d \tau\right] N_{\delta}(t) d t$.

Now we may apply a method of successive approximations and integrate the integro-differential equations. I have already applied the method of successive approximations to the resolution of the integro-differential equations relative to the historical case for two species; these equations are a particular case $(n=2)$ of those now found.

The procedure for the application of the method of successive approximations to the case when $n=2$ has been exposed in detail in the work "Leģons sur la théorie mathématique de la lutte pour la vie" (see page 192 and following). I think it unnecessary to explain here the easy extension of this proceedure to the general case.

10. There are many interesting particular cases to examine. For instance if the actions are reciprocal (as in the case in which the individuals of the various species devour each other) it will be convenient to assume $A_{s r}, A_{r s}$, with different signs, and so $F_{s r}, F_{r s}$. Some of these may be zero.

If we admit both for the $A_{s r}, A_{r s}$ and for the $F_{s r}, F_{r s}$, that they will be in certain cases inversely proportional to the values

$$
\beta_{1}, \beta_{2}, \ldots \beta_{n}
$$

of the individuals, we shall put

$$
\begin{aligned}
A_{s r} & =\frac{a_{s r}}{\beta_{r}}, \quad A_{r s}=\frac{a_{r s}}{\beta_{s}}, \quad a_{s r}=-a_{r s}, \\
F_{s r}(t-\tau) & =\frac{f_{s r}(t-\tau)}{\beta_{r}}, \quad F_{r \delta}(t-\tau)=\frac{f_{r s}(t-\tau)}{\beta_{8}}, \quad f_{s r}=-f_{r s} ;
\end{aligned}
$$

so that the equations $\left(B^{\prime \prime \prime}\right)$ become

(B) $\left.B^{i v}\right) \quad \beta_{r} \frac{d N_{r}}{d t}=\left\{\beta_{r} \epsilon_{r}+\sum_{1}^{n}\left[a_{s r} N_{s}(t)+\int_{0}^{T_{v}} N_{s}(t-\tau) f_{8 r}(\tau) d \tau\right]\right\} N_{r}(t)$. 
The equations which give the stationary states (conditions of equilibrium) will then be

when we put

$$
\beta_{r} \epsilon_{r}+\sum_{1}^{n} c_{s r} K_{s}=0
$$

From $\left(C^{\prime}\right)$ we deduce

$$
C_{s r}=\frac{c_{s r}}{\beta_{r}}, \quad c_{s r}=-c_{r s}
$$

$$
\sum_{1}^{n} \beta_{r} \epsilon_{r} K_{r}=0
$$

from which we may deduce, for the historical case, the same laws of reciprocity that we have enunciated in previous works in the case of immediate actions. (See the conference mentioned in §2.)

It is however necessary to observe that while in the last case the populations of equilibrium coincide with the averages of the populations, we cannot say the same, at least in general, in the historical case.

11. We confine ourselves, in the present short essay, to the preceding general considerations and leave aside any further developments.

Finally we consider it convenient to summarise the following conclusions:

I. That with conveniently appropriate hypotheses the general case of historical actions, already studied for two species, can also be examined for any number of species.

II. That in this case we can obtain some general integro-differential equations which include all those previously given for the problems of biological struggle.

III. That we can completely and easily integrate these equations with simple methods of successive approximations.

IV. That we can undertake to study the stationary case.

Via in Lucina 17

Roma, Italy. 